\title{
Therapie epithelialer Tumoren *
}

\author{
Treatment of Epithelial Tumours
}

Autoren

Institut

\section{Terhorst, E. Stockfleth}

Klinik für Dermatologie, Venerologie und Allergologie, Charité Universitätsmedizin Berlin

\section{Bibliografie}

DOI $10.1055 / \mathrm{s}-2008-1077567$

Akt Dermatol 2008; 34:

482-486 @ Georg Thieme

Verlag KG Stuttgart · New York ISSN 0340-2541

Korrespondenzadresse

Prof. Dr. med.

Eggert Stockfleth

Hauttumorcentrum Charité

Klinik für Dermatologie,

Venerologie und Allergologie

Charité Universitätsmedizin

Charitéplatz 1

10117 Berlin

eggert.stockfleth@charite.de

\section{Zusammenfassung \\ $\nabla$}

Weltweit ist eine Zunahme der Inzidenz der epithelialen Hauttumoren um $8 \%$ jährlich zu vermerken. Die häufigsten Vertreter stellen die aktinische Keratose, das Basalzellkarzinom und das Plattenepithelkarzinom dar. UV-Exposition gilt als der wichtigste Risikofaktor, so entstehen epitheliale maligne Tumoren meist auf chronisch sonnenexponierter Haut (Gesicht, Kopfhaut, Handrücken) als Folge kumulativer UV-Schädigung. Eine chronisch lichtexponierte Region weist häufig transformierte Keratinozyten in verschiedenen Graduierungen, einschließlich subklinischer Läsionen, auf. Topische Therapieformen wie Imiquimod, Diclofenac-Hyaluronsäure und die photodynamische Therapie werden die-

\section{Einleitung \\ $\nabla$}

Weltweit ist eine Zunahme der Inzidenz der epithelialen Hauttumoren um $8 \%$ jährlich zu vermerken. Die häufigsten Vertreter stellen die aktinische Keratose, das Basalzellkarzinom und das Plattenepithelkarzinom dar. Schätzungen gehen davon aus, dass in der Zukunft ungefähr jeder zweite Deutsche in seinem Leben an aktinischen Keratosen erkranken wird. Ähnlich hoch sind die Schätzungen für das Basalzellkarzinom, welches etwa jeder Dritte im Lebensverlauf entwickeln wird [1].

UV-Exposition gilt als der wichtigste Risikofaktor für die Entstehung von malignen Hauttumoren, so entstehen epitheliale maligne Tumoren meist auf chronisch sonnenexponierter Haut (Gesicht,

\footnotetext{
* Nach einem Vortrag gehalten anlässlich des Symposions „Moderne Aspekte der Dermatoonkologie“ organisiert vom Tumorzentrum und der Klinik für Dermatologie, Venerologie und Allergologie - Immunologisches Zentrum, Städtisches Klinikum Dessau, März 2007.
}

ser Feldkanzerisierung unterschiedlich gerecht. Eine rechtzeitige Diagnosestellung, zum Beispiel im Rahmen des Hautkrebs-Screenings, ermöglicht den betroffenen Patienten von diesen neuen, nicht invasiven Therapieformen zu profitieren. Bei solitären Läsionen kommen weiterhin die Kryotherapie, Kürettage, Lasertherapie und in Einzelfällen die Röntgen-Weichstrahltherapie zum Einsatz. Mittel der Wahl beim Plattenepithelkarzinom und bei bestimmten Fällen des Basalzellkarzinoms ist die mikrografisch kontrollierte Exzision. Ein konsequenter präventiver Lichtschutz mit spezifischen Verhaltensänderungen, textiler Bedeckung und der Verwendung von UV-Schutzpräparaten stellt die wichtigste langfristige medizinische Intervention dar.

Kopfhaut, Handrücken) als Folge kumulativer UV-Schädigung. Daneben stellen heller Hauttyp (I und II), aber auch genetische Faktoren weitere Risikofaktoren dar. Neben einer vermehrten Sonnenexposition der Bevölkerung ist die demografische Überalterung der Bevölkerung ein weiterer Grund für die steigende Inzidenz epithelialer Tumoren. Epitheliale Tumoren präsentieren somit ein enormes medizinisches Problem, durch das gesteigerte Kosten für das Gesundheitssystem entstehen. Neben einer frühen Diagnosestellung bedeutet der präventive intensive Sonnenschutz mit spezifischen Verhaltensänderungen, textilem Schutz und der Verwendung von UV-Schutzpräparaten eine wichtige dermatologische Intervention [2].

Die kutane Tumordiagnostik basiert auf der klinischen Untersuchung, einer genauen Anamnese und der histologischen Analyse. Als unterstützendes Verfahren hat sich in den letzten 20 Jahren die Dermatoskopie etabliert. Innovative Methoden zur nicht-invasiven Diagnostik von Hauttumoren werden in den letzten Jahren vermehrt 


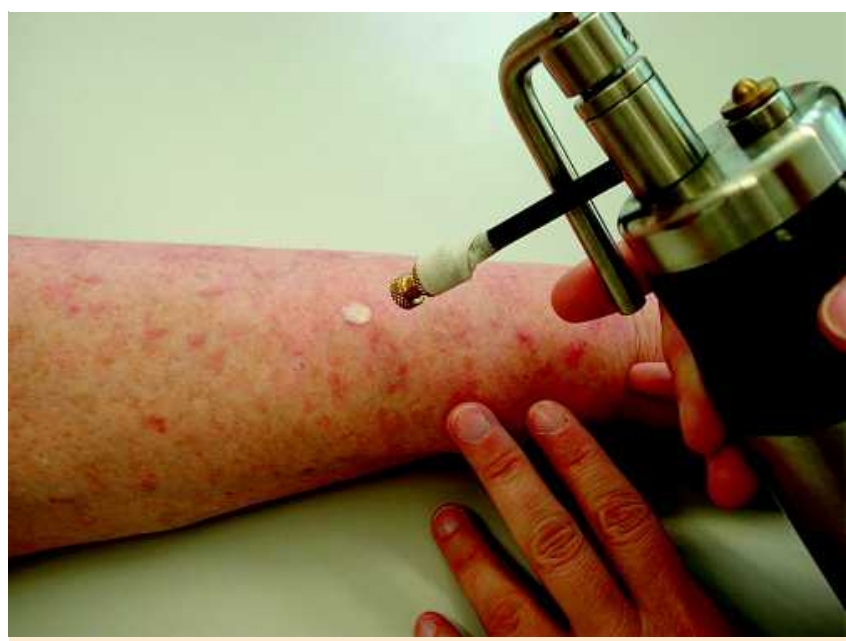

Abb. 1 Kryotherapie mit flüssigem Stickstoff (Sprayverfahren) am Unterarm einer 67-jährigen Patientin.

evaluiert. Besonders die konfokale Mikroskopie erlaubt eine Differenzierung epithelialer Tumore und kann speziell zur Definition der vertikalen Tumorausdehnung herangezogen werden [3]. Bei invasiven Plattenepithelkarzinomen (ab pT2) sollte die Abklärung einer Lymphknotenbeteiligung mithilfe bildgebender Verfahren erfolgen, und kann unter Umständen auch die Durchführung einer Szintigrafie und eines CT beinhalten. Bei Basalzellkarzinomen und in-situ Plattenepithelkarzinomen sind weiterführende Untersuchungen nicht routinemäßig indiziert, da nicht von einer Metastasierung ausgegangen werden kann.

Aufgrund der Aufklärung der molekularen Mechanismen, die den verschiedenen kutanen Tumoren zugrunde liegen, konnten in den letzten Jahren neue, spezifische, auf die Pathogenese abgestimmte Therapien entwickelt werden. Die Hoffnung besteht, dass im Rahmen des neu etablierten Hautkrebs-Screening Patienten mit epithelialen Tumoren möglichst frühzeitig erkannt werden. Diese Patienten könnten dann zum Beispiel von den neuen topischen Therapien profitieren.

\section{Basalzellkarzinom}

Basalzellkarzinome sind langsam wachsende, maligne, epitheliale Hauttumoren, die meist in lichtexponierten Hautarealen auftreten. Bei extrem geringer Metastasierungstendenz findet sich ein lokal invasives, destruierendes Wachstum. Charakteristischerweise zeigen sie ein Differenzierungsspektrum ähnlich der Haaranlage. Auf molekularbiologischer Ebene finden sich teilweise Mutationen im Patched-Gen sowie in Genen, die am sonic-hedgehog-Signaltransduktionsweg beteiligt sind. Die unterschiedlichen klinischen Erscheinungsformen korrelieren mit einem ebenfalls unterschiedlich agressiven Wachstumsverhalten.

Therapeutisches Ziel ist immer die sichere und vollständige Behandlung des Basalzellkarzinoms mit dem besten funktionellkosmetischen Ergebnis. Die chirurgische Exzision stellt eine hocheffektive Therapieform für primäre Basalzellkarzinome dar. Für größere, sklerodermiforme und infiltrative Basalzellkarzinome eignet sich insbesondere die mikrografisch kontrollierte Exzision nach Mohs [4].

Bei ausreichender Erfahrung lassen sich mit Kryotherapie, Kürettage und Elektrokaustik bei kleinen, gut differenzierten Basal-
Tab. 1 Imiquimod.

Indikation
Superfizielle Basalzellkarzinome
Aktinische Keratosen
(Gesicht, Kopfhaut)

\section{Anwendung}

Fünfmal/Woche, 6 Wochen Dreimal/Woche, zunächst 4 Wochen lang, dann 4 Wochen Pause, nach Evaluierung ggfs. erneut 4 Wochen

Anwendungshinweise: Die Creme sollte vor dem Zubettgehen auf die gereinigte Haut aufgetragen werden, acht Stunden belassen und dann abgewaschen werden. Es kann zu deutlichen, gewünschten Entzündungen an den behandelten Stellen kommen.

zellkarzinomen in unkritischer Lokalisation gute Heilungsraten erreichen, nicht geeignet sind diese Therapieverfahren jedoch bei größeren, rezidivierten, als Risikotumoren klassifizierten Basalzellkarzinomen [5] (siehe $\bullet$ Abb. 1).

Die Radiotherapie ist eine früher weiter verbreitete Methode, die noch immer in einigen Fällen sehr hilfreich ist. Einschränkende Nebenwirkungen stellen die karzinogene Wirkung sowie die sich entwickelnden Teleangiektasien, Atrophie und Fibrose dar. Imiquimod gehört zu der Substanzklasse der sogenannten Immune Response Modifier (IRM). Imiquimod induziert nach Rezeptorbindung an den toll-like receptor 7 eine Reihe von Zytokinen, die vor allem die zelluläre Immunität (Th1) steigern und dadurch antivirale und antitumorale Eigenschaft besitzen (siehe - Tab. 1).

Bei superfiziellen Basalzellkarzinomen ließen sich in histologisch kontrollierten Studien unter täglicher Applikation von 5\% Imiquimod-Creme (Aldara ${ }^{\circledR}$ ) für 6 Wochen komplette Abheilungsraten von 87,9\% erzielen. Unter der derzeit empfohlenen Dosierung von 5 Anwendungen pro Woche über 6 Wochen sind $82 \%$ komplette Abheilungsraten beschrieben. Zwischenresultate einer 5-Jahres-Studie zur Behandlung von superfiziellen Basalzellkarzinomen zeigen 12 Wochen nach Therapie eine $90 \%$ ige Abheilung und nach 24 Monaten immerhin noch 79,4\% Rezidivfreiheit. Bei nodulären Basalzellkarzinomen konnten unter täglicher Applikation von Imiquimod nach 6 Wochen Therapie 71\% und nach 12 Wochen Applikation 76\% Abheilung erreicht werden. Die Anwendung von Imiquimod bei nodulären Basalzellkarzinomen wird derzeit nicht empfohlen [6].

Nach Anwendung der photodynamischen Therapie (PDT) konnten in Studien komplette Abheilungsraten von bis zu $88 \%$ erzielt werden. Langfristige Daten über Rezidivraten stehen noch aus, sodass eine abschließende Bewertung der Rolle der PDT in der Behandlung von Basalzellkarzinomen nicht möglich ist [1].

Zur Behandlung inoperabler sowie der extrem seltenen metastasierten Basalzellkarzinome wird eine Chemotherapie mit Cisplatin $75 \mathrm{mg} / \mathrm{m}^{2}$ plus Doxorubicin $50 \mathrm{mg} / \mathrm{m}^{2}$ eingesetzt.

Eine orale Retinoidtherapie wird in der Prävention neuer Basalzellkarzinome, z.B. im Rahmen des Basalzell-Naevus-Syndroms eingesetzt. Probleme stellen die hohen erforderlichen Dosierungen und nach Therapie auftretenden Rezidive dar [1].

\section{Aktinische Keratose \\ $\nabla$}

Die aktinische Keratose ist ein Carcinoma in situ und somit eine Frühform des invasiven Plattenepithelkarzinoms. Es erfüllt alle Kriterien eines Plattenepithelkarzinoms, bleibt jedoch auf die Epidermis beschränkt, ohne die Basalmembran zu durchbrechen. Zu diesen Kriterien gehören atypische Keratinozyten, eine 
hohe Mutationsrate des Tumorsuppressorgens p53 und charakteristische chromosomale Aberrationen [7]. Etwa 10\% der aktinischen Keratosen gehen in ein invasives Plattenepithelkarzinom über. Bisher sind keine eindeutigen Kriterien definiert, mit deren Hilfe man vorhersagen könnte, welche aktinische Keratose invasiv wachsen wird. Deswegen gilt es, alle aktinischen Keratosen zu behandeln. Bei immunsupprimierten Patienten, v.a. Organtransplantatempfängern, liegt eine höhere Progressionsrate von aktinischen Keratosen zu invasiven Plattenepithelkarzinomen von etwa $30 \%$ vor [8].

Eine Vielzahl unterschiedlicher Behandlungsverfahren steht zur Therapie der aktinischen Keratose zur Verfügung. Es kann sinnvoll sein, suspekte Läsionen zu exzidieren oder mit einer kleinen Hautbiopsie zu untersuchen.

Die Kryotherapie ist ein häufig eingesetztes, lokal destruierendes Behandlungsverfahren. Die Kryotherapie ist bezüglich Dauer, Intensität und Häufigkeit nicht standardisiert und so therapeutenabhängig. Mögliche Folgeerscheinungen sind Narbenbildung, Hypo- und Hyperpigmentierung. Die Ansprechraten reichen von 75 bis 98 Prozent, die Rezidivraten erreichen bis zu 43 Prozent innerhalb eines 1-Jahres-Nachbeobachtungszeitraums [9].

Die Kürettage mit oder ohne Elektrodesikkation wird insbesondere bei ausgeprägt hyperkeratotischen Veränderungen erwogen, nach Biopsie und bei Versagen anderer Behandlungsmodalitäten.

Der $\mathrm{CO}_{2}$ - und Erbium-YAG-Laser stehen zur Abtragung aktinischer Keratosen zur Verfügung. Nebenerscheinungen sind Schmerzen, lokale Entzündungen, Pigmentierungsstörungen und Narbenbildung. In 90 bis 100 Prozent ist ein vollständiges Entfernen der aktinischen Keratosen dokumentiert, Rezidivraten liegen bei etwa 10 bis 15 Prozent für Einzelläsionen 3 bis 6 Monate nach Behandlung [1].

Die Röntgen-Weichstrahltherapie wird wegen seines karzinogenen Effektes bei aktinischen Keratosen als obsolet angesehen.

Beim chemischen Peeling wird mit kaustisch wirkenden Substanzen wie Trichloressigsäure, hochprozentiger Alpha-Hydroxy-Säure oder Phenolzubereitungen Gewebe zerstört. Chemisches Peeling ist für die Therapie der aktinischen Keratosen nicht zugelassen. Mögliche Nebenwirkungen sind Schmerzen, Narbenbildung und Entzündungen. Die Reduktion der aktinischen Keratosen wird mit etwa 75 Prozent angegeben, Rezidivraten mit 25 Prozent innerhalb eines Jahres nach Therapie [10]. Eine chronisch lichtexponierte Region weist häufig transformierte Keratinozyten in verschiedenen Graduierungen, einschließlich subklinischer Läsionen, auf. Dies war der Grund, den Begriff Feldkanzerisierung oder field cancerisation einzuführen [10]. Topische Therapieformen wie die photodynamische Therapie, Diclofenac-Hyaluronsäure, 5-Fluorouracil und Imiquimod werden dieser Feldkanzerisierung unterschiedlich gerecht.

Für die photodynamische Therapie hat die European Medical Evaluation Agency (EMEA) die Substanz MAOP (Handelsname Metvix Creme ${ }^{\circledR}$ ) in Kombination mit einer geeigneten Lichtquelle $\left(\right.$ Aktilite $^{\circledR}$ ) für die Indikation aktinische Keratose zugelassen (siehe Abb. 2 u. 3). Hauptnebenwirkung der PDT ist der lokale Schmerz bei der Bestrahlung, Die klinischen Daten zeigen eine komplette Abheilung bei 70 bis 78 Prozent nach einer Anwendung, 90 Prozent Abheilung nach einer wiederholten Anwendung eine Woche später [11].

Zur lokalen Therapie bei aktinischen Keratosen steht Diclofenac als 3-prozentiges Gel in 2,5 Prozent Hyaluronsäure mit dem Präparat Solaraze Gel ${ }^{\circledR}$ (Fa. Almirall-Hermal) zur Verfügung, wel-

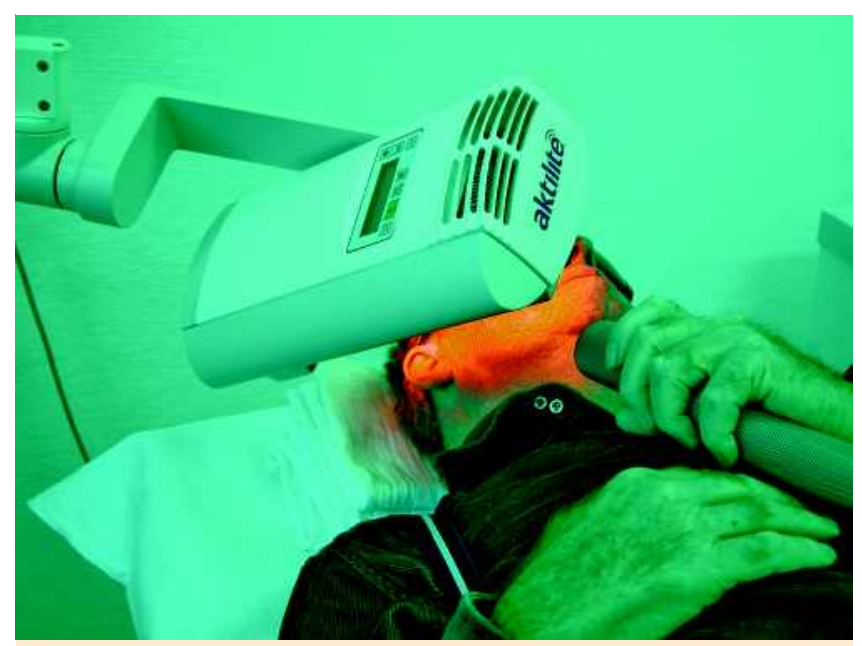

Abb. 2 Anwendung von photodynamischer Therapie bei therapieresistenten aktinischen Keratosen.

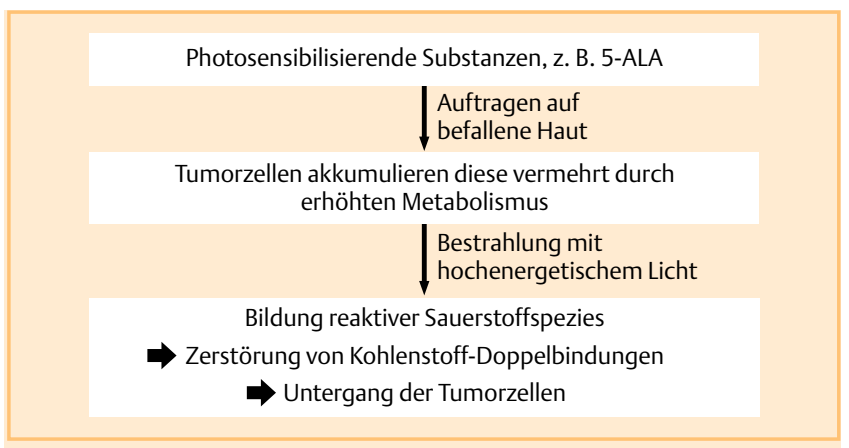

Abb. 3 Wirkmechanismus der photodynamischen Therapie.

Tab. 2 Diclofenac- Hyaluronsäuregel.

\begin{tabular}{|ll}
\hline Indikation & Aktinische Keratosen \\
\hline Anwendung & Zweimal täglich für 3-4 Monate \\
\hline Wirkmechanismus & Diclofenac hemmt sowohl die Cyclooxygenase \\
& 1 wie auch 2. COX 2 begünstigt die Karzinoge- \\
& nese der Haut durch Förderung der Proliferation \\
& und Neoangiogenese sowie Hemmung der \\
& Apoptose. \\
& Die Hyaluronsäure führt vermutlich zu einer \\
& besseren Bioverfügbarkeit am Wirkort und \\
& möglicherweise auch zu einer verbesserten \\
& Penetration des Diclofenacs durch die obere \\
& Epidermis.
\end{tabular}

ches in Europa und den USA zur Behandlung aktinischer Keratosen zugelassen ist (siehe $\bullet$ Tab. 2, $\bullet$ Abb. 4). Die Anwendung erfolgt zweimal täglich über 3 bis 4 Monate. Mögliche Nebenwirkungen sind lokale Irritationen. Bei Therapie über 60 Tage zeigten sich Ansprechraten von 70 Prozent [12]. Eine komplette Reduktion der aktinischen Keratosen wiesen 50 Prozent der Patienten nach 90-tägiger Anwendung von Diclofenac 3 Prozent in Hyaluronsäure auf [13].

Die 5-prozentige 5-Fluorouracil-Salbe wird zweimal täglich, üblicherweise über zwei bis vier Wochen auf die betroffenen Hautstellen aufgetragen (siehe Abb.5). Starke Irritationen und Hautentzündungen sind eine regelmäßige Nebenwirkung. Nach Therapie über vier Wochen präsentierten 96 Prozent der Patienten eine initiale klinische Heilung, die bei 67 Prozent histolo- 


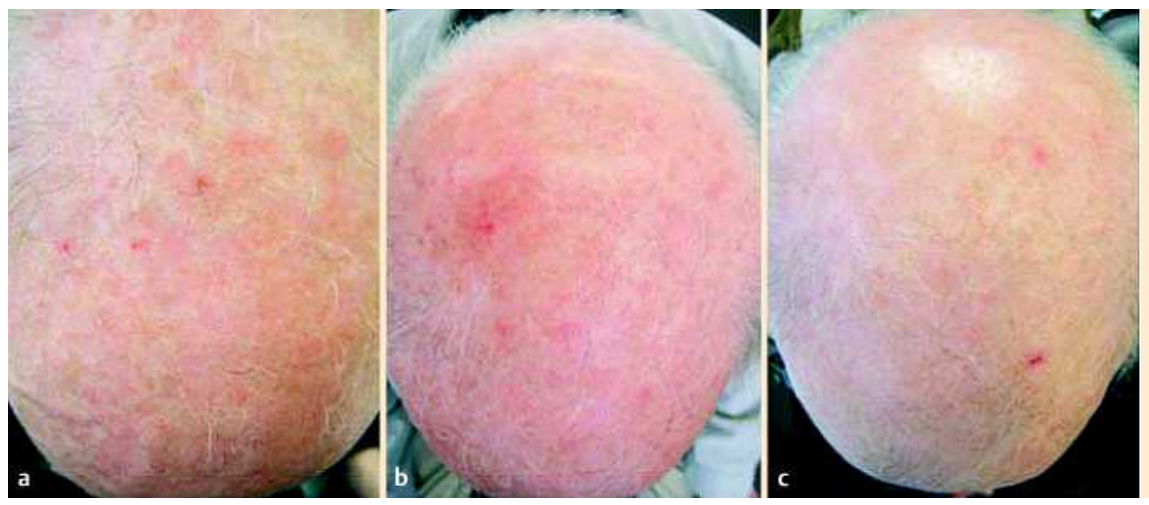

Abb. 4 Therapie aktinischer Keratosen am Kapillitium mit Solaraze Gel ${ }^{\circledR}$ über 10 Wochen. a vor der Therapie. $\mathbf{b} 8$ Wochen Behandlung mit Solaraze Gel ${ }^{\circledR}$. c 2 Wochen nach 10-wöchiger Solaraze Ge ${ }^{\circledR}$-Behandlung. Die aktinischen Keratosen sind hier komplett abgeheilt.
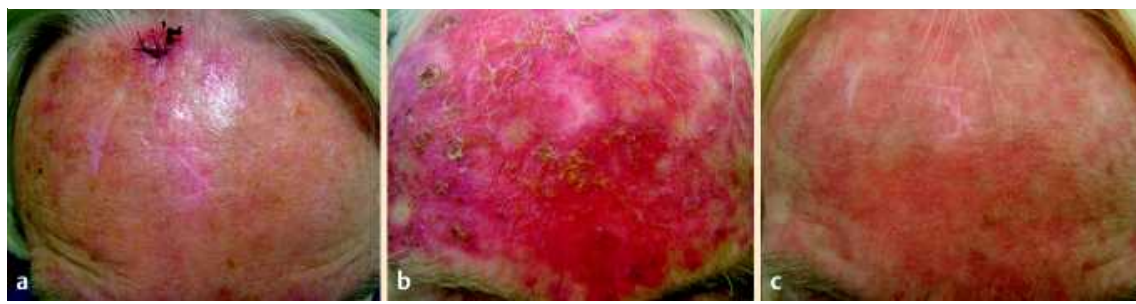

Abb. 5 Therapie aktinischer Keratosen am Kapillitium mit 5-Fluorouracil Creme. a vor der Therapie. b 1 Woche unter Behandlung. c 2 Wochen nach Behandlung. Die Bilder im Verlauf zeigen die typische starke Rötung und Entzündung. Die aktinischen Keratosen sind hierunter nahezu vollständig abgeheilt.
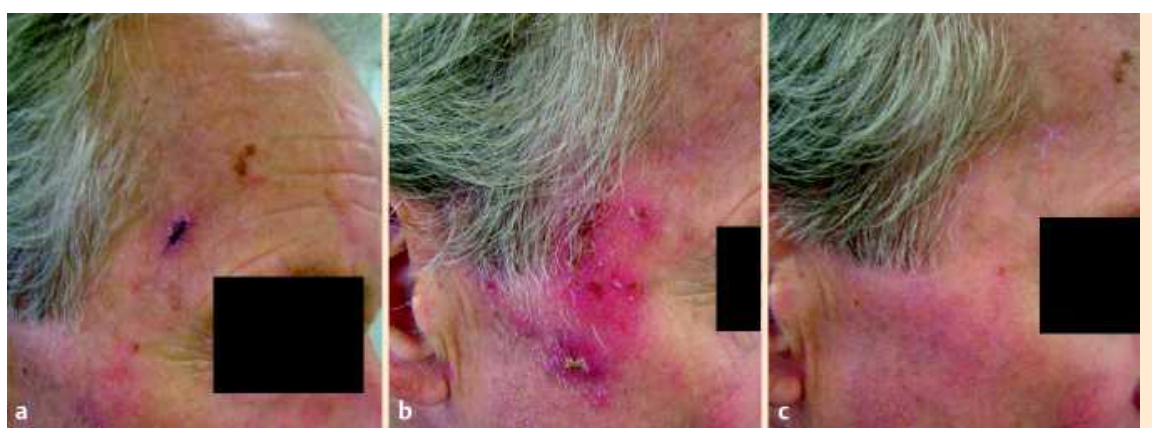

Abb. 6 Therapie aktinischer Keratosen an der Schläfe mit Aldara $5 \%$ Creme $^{\circledR}$. a vor der Therapie. b 4 Wochen unter Behandlung. $c 4$ Wochen nach 4-wöchiger Aldara $5 \%$ Creme $^{\circledR}$ Therapie. Die aktinischen Keratosen heilten unter der erwünschten vorübergehenden lokalen Entzündungsreaktion komplett ab.

gisch bestätigt wurde. Bei 46 Prozent der zunächst abgeheilten aktinischen Keratosen entwickelten sich Rezidive, lediglich 33 Prozent der Patienten bildeten in dem Nachbeobachtungszeitraum von 12 Monaten keine neuen Läsionen in dem behandelten Areal [9].

Imiquimod wird als Aldara $5 \%$ Creme ${ }^{\circledR}$ bei aktinischen Keratosen dreimal die Woche zunächst für vier Wochen angewandt (siehe - Abb.6). Wenn nach einer 4-wöchigen Pause nicht alle Läsionen verschwunden sind, wird die Therapie eventuell für weitere 4 Wochen fortgeführt (siehe $\bullet$ Tab. 1). Unerwünschte Wirkungen sind Rötung, Juckreiz, Schmerzen, Brennen, Erosion und Krustenbildung. Beschrieben sind auch systemische Nebenwirkungen wie eine grippeähnliche Symptomatik.

Eine Studie mit 829 Patienten zeigte eine komplette Clearancerate von 68,9 Prozent, zu einer Reduktion von mindestens 75 Prozent kam es bei 80,2 Prozent der Patienten [14].

\section{Bowen}

$\nabla$

Der M. Bowen ist ein Carcinoma in situ der Haut, die entsprechende Veränderung der Schleimhaut wird als Erythroplasie Queyrat bezeichnet. Unbehandelt können beide in ein invasives Karzinom, ein Plattenepithelkarzinom vom Bowentyp, übergehen.

Neben der chirurgischen Entfernung kommen zur Behandlung des M. Bowen und der Erythroplasie Queyrat auch die Kryothe- rapie, ablative Lasertherapie und die lokale Chemotherapie mit 5-FU oder Imiquimod infrage [15].

\section{Plattenepithelkarzinom \\ $\nabla$}

Plattenepithelkarzinome beginnen intraepithelial als Carcinoma in situ (aktinische Keratosen, Morbus Bowen) und gehen nach unterschiedlicher Zeit in invasiv und destruktiv wachsende Tumoren über. Plattenepithelkarzinome der Haut treten häufig an den Übergängen von Haut zu Schleimhaut und an den Schleimhäuten selbst auf.

Die Exzision und histologische Kontrolle von Exzisionsrand und Wundgrund stellt das Standardvorgehen in der lokalen Behandlung dar. Wegen der eher seltenen Metastasierung und dem hohen Durchschnittsalter der Patienten wird nur bei Hochrisikopatienten, z.B. Organtransplantatempfängern, eine Sentinellymphknotenbiopsie durchgeführt. Mit relativ hoher Sicherheit (88-96\%) wird eine dauerhafte lokale Heilung durch die mikrographische Chirurgie erreicht, d.h. die sparsame chirurgische Exzision mit einer nachvollziehbaren Markierung und anschließender lückenloser Histologie der Exzisatschnittränder. Beim desmoplastischen Typ bedarf es noch einer zusätzlichen Sicherheitsnachresektion von ca. $5 \mathrm{~mm}$ über die festgestellten tumorfreien Schnittränder hinaus. Bei stichprobenartiger histologischer Kontrolle im Rahmen der konventionellen Chirurgie muss wegen der lokalen Infiltration der Plattenepithelkarzinome der 
Haut mit einem etwas höheren Rezidivrisiko gerechnet werden (5-10\%) [16].

Behandlungsmöglichkeiten ohne histologische Kontrolle wie Röntgenbestrahlung, Kryotherapie, photodynamische Therapie, Laserbehandlung kommen nur bei inoperablen Tumoren infrage [16].

Bei Plattenepithelkarzinomen im Stadium III und IV kommt unter Berücksichtigung des Allgemeinzustands eine Polychemotherapie mit Bleomycin, Cisplatin, 5-Fluorouracil oder hoch dosiertem Methotrexat zum Einsatz. Die Ansprechraten erreichen bis zu $80 \%$, die Therapie ist aber nicht kurativ und die Patienten erleiden in der Regel ein Rezidiv. Da bis jetzt kein Standardschema existiert, sollte eine Chemo- oder Immuntherapie des metastasierten Plattenepithelkarzinoms der Haut möglichst in Studien erfolgen [1].

\section{UV-Schutz und Prävention \\ $\nabla$}

UV-Exposition ist ein wesentlicher Faktor für die Entstehung von Hautkrebs. Ziel eines konsequenten UV-Schutzes ist die Verhinderung akuter Lichtschädigung der Haut, der frühzeitigen Hautalterung sowie einer Minimierung des Erkrankungsrisikos für UV-induzierte Hautkrebsformen. Neben einer Expositionsprophylaxe stellen die textilen Sonnenschutzmaßnahmen und die Verwendung von Sonnenschutzmitteln mit breitem UVA- und UVB-Absorbtionsspektrums die wichtigsten Maßnahmen dar.

In einer kürzlich veröffentlichten Studie wurden 60 organtransplantierte Patienten mit täglicher Applikation von hochprotektivem, liposomalen Sonnenschutz (Daylong actinica ${ }^{\circledR}$, Fa. Spirig) mit einer Kontrollgruppe verglichen. Im Laufe der Studiendauer von 24 Monaten, entwickelten 42 von 120 Patienten 83 neue aktinische Keratosen (alle in der Kontrollgruppe). 8 Patienten der Kontrollgruppe entwickelten neue Plattenepithelkarzinome, im Vergleich zu keinen in der Sonnenschutzgruppe. In der Gruppe der Patienten, die Sonnenschutz verwendet haben, haben sich 102 Läsionen aktinischer Keratosen spontan zurückgebildet. Sonnenschutzmaßnahmen haben also einen positiven Einfluss in der Hochrisikogruppe von Organtransplantatempfängern [8].

\section{Abstract}

\section{Treatment of Epithelial Tumours \\ $\nabla$}

The incidence of epithelial skin cancers increases worldwide by $8 \%$ annually. The most important representatives are actinic keratoses, basal cell carcinoma and squamous cell carcinoma. Exposure to UV-rays is considered as the fundamental risk factor, epithelial malignant tumours thus arise on chronically sun-exposed areas of the skin as face, scalp and back of the hand. These sun-exposed areas on the skin often show multiple lesions of transformed keratinocytes in different gradings, including subclinical lesions. Topical therapies as imiquimod, diclofenac-hyaluronic acid and photodynamic therapy give variable considera- tion to this idea of field cancerisation. An early diagnosis enables patients to benefit from these new, non-invasive therapies. Single lesions can also be cured with cryosurgery, curettage, laser therapy and in exceptional cases with radiotherapy. Standard of care for the squamous cell carcinoma and certain forms of basal cell carcinoma is Moh's surgery. A consequent preventive light shield with changes of behaviour, textile shields and the use of sunscreen is the most important long-term intervention.

\section{Literatur}

1 Stockfleth E. Hautkrebs - ein Leitfaden für die Praxis. 2. Auflage. Bremen: UNI-MED, 2008

2 Green A, Williams G, Neale R et al. Daily sunscreen application and betacarotene supplementation in prevention of basal-cell and squamous-cell carcinomas of the skin: a randomised controlled trial. Lancet 1999; 354: $723-729$

3 Ulrich M, Stockfleth E, Roewert-Huber J, Astner S. Noninvasive diagnostic tools for nonmelanoma skin cancer. Br J Dermatol 2007; 157 (Suppl 2): 56 - 58. Review.

4 Telfer NR, Colver GB, Morton CA, British Association of Dermatologists. Guidelines for the management of basal cell carcinoma. $\mathrm{Br} J$ Dermatol 2008; 159: $35-48$

5 Nordin P, Larkö O, Stenquist B. Five-year results of curettage-cryosurgery of selected large primary basal cell carcinomas on the nose: an alternative treatment in a geographical area underserved by Mohs' surgery. Br J Dermatol 1997; 136: 180 - 183

6 Sterry W, Ruzicka T, Herrera E, Takwale A, Bichel J, Andres K, Ding L, Thissen MR. Imiquimod 5\% cream for the treatment of superficial and nodular basal cell carcinoma: randomized studies comparing low-frequency dosing with and without occlusion. Br J Dermatol 2002; 147: $1227-1236$

7 Ackerman $A B$, Mones JM. Solar (actinic) keratosis is squamous cell carcinoma. Br J Dermatol 2006; 155: 9-22

8 Ulrich C, Degen A, Patel MJ, Stockfleth E. Sunscreens in organ transplant patients. Nephrol Dial Transplant 2008; 23: 1805-1808

9 Krawtchenko N, Roewert-Huber J, Ulrich M, Mann I, Sterry W, Stockfleth $E$. A randomised study of topical $5 \%$ imiquimod vs. topical 5-fluorouracil vs. cryosurgery in immunocompetent patients with actinic keratoses: a comparison of clinical and histological outcomes including 1-year follow-up. Br J Dermatol 2007; 157 (Suppl 2): 34-40

10 Stockfleth E, Kerl H, Guideline Subcommittee of the European Dermatology Forum. Guidelines for the management of actinic keratoses. Eur J Dermatol 2006; 16: 599-606

11 de Berker D, McGregor JM, Hughes BR, British Association of Dermatologists Therapy Guidelines and Audit Subcommittee. Guidelines for the management of actinic keratoses. Br J Dermatol 2007; 156: 222 230. Erratum in: Br J Dermatol 2008; 158:873

12 Rivers JK, Arlette J, Shear $N$ et al. Topical treatment of actinic keratoses with 3,0\% diclofenac in 2,5\% hyaluronan gel. Br J Dermatol 2002; 146: $94-100$

13 Wolf JR, Taylor JR, Tschen E. Topical 3\% diclofenac in 2Æ 5\% hyaluronan gel in the treatment of actinic keratoses. Int J Dermatol 2001; 40: $709-713$

14 Stockfleth E, Sterry W, Carey-Yard M, Bichel J. Multicentre, open-label study using imiquimod $5 \%$ cream in one or two 4-week courses of treatment for multiple actinic keratoses on the head. $\mathrm{Br} J$ Dermatol 2007; 157: $41-46$

15 Thestrup-Pedersen K, Ravnborg L, Reymann F. Morbus Bowen. A description of the disease in 617 patients. Acta Derm Venereol 1988; 68: 236-239

16 Breuninger H, Sebastian G, Kortmann RD, Wolff K, Bootz F, Garbe C. [Brief guidelines: squamous cell carcinoma of the skin, lip and eyelids]. J Dtsch Dermatol Ges 2006; 4: 260-262 